\title{
AUTOMATIC LOCALIZATION OF INDOOR SOCCER PLAYERS FROM MULTIPLE CAMERAS*
}

\author{
Erikson Freitas de Morais ${ }^{1}$, Siome Goldenstein ${ }^{1}$ and Anderson Rocha ${ }^{1}$ \\ ${ }^{1}$ Institute of Computing, University of Campinas, Campinas, Brazil \\ \{emorais, siome, anderson.rocha\}@ic.unicamp.br
}

\author{
Keywords: \\ Indoor Soccer; Sports Automation; Multiple Camera Observations
}

\begin{abstract}
:
Nowadays, there is an ever growing quest for finding sophisticated performance evaluation tools by team sports that could give them an additional inch or a quarter of a second of advantage in a competition. Using cameras to shoot the events of a game, for instance, the teams can analyze the performance of the athletes and even extrapolate the data to obtain semantical information about the behavior of the teams themselves at relatively low costs. In this context, this paper introduces a new approach for better estimating the positions of indoor soccer players using multiple cameras at all moments of a game. The setup consists of four stationary cameras set around the soccer court. Our solution relies on individual object detectors (one per camera) working in the image coordinates and a robust fusion approach working in the world coordinates in a plane that represents the soccer court. The fusion approach relies on a gradient ascent algorithm over a multimodal bidimensional mixture of Gaussians function representing all the players in the soccer court. In the experiments, we show that the proposed solution improves standard object detector approaches and greatly reduces the mean error rate of soccer player detection to a few centimeters with respect to the actual positions of the players.
\end{abstract}

\section{Introduction}

With the popularization and low cost of camcorders, the shooting of entire games involving team sports has become an important aid to coaches and the technical staff of a team. A game shooting contains all the athletes' correct and wrong moves on a given game. A specialized staff team can evaluate and annotate the videos to classify the most important moves of interest. For instance, after annotating the events of a game it would be possible to: plot charts depicting the number of missed passes by team or individual player; view correctly executed moves and also the badly executed ones; or even point out bad positioning during the game. Such annotations feed the technical committee with invaluable information for improving the team for future matches.

The use of camcorders to record team sports

${ }^{*}$ We thank the financial support of Microsoft Research, FAPESP and CNPq (Grant 141054/2010-7). games can be greatly enhanced by the use of multiple cameras under different points of view. Missing information in the point of view of a given camera can be compensated by other views in different cameras. The redundant information can also help to better estimate the measures and achieve more reliable conclusions regarding the games.

In this context, this paper's objective is to estimate the positions of indoor soccer players at every moment of a given game using multiple cameras. This task is the first one towards more sophisticated trajectory analysis for each individual player.

The problem we address in this paper consists of a setup with four cameras around an indoor soccer court. Figure 1 shows a frame under each point of view while Figure 2 depicts the camera positioning around the soccer court and the different points of view.

To deal with this problem, all the information we have consist of the captured videos and 


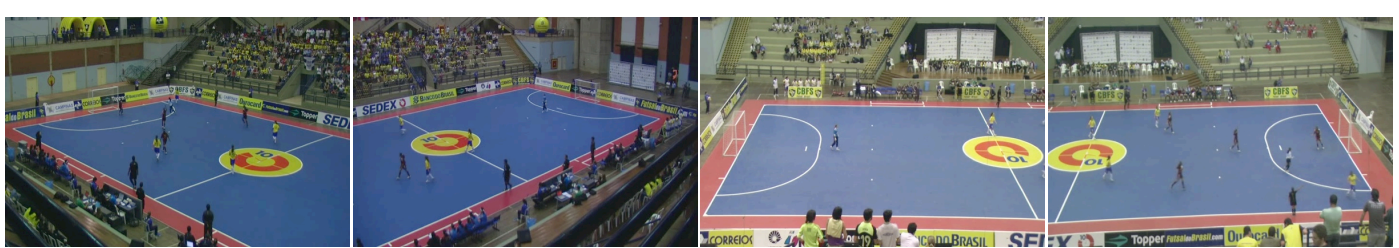

Figure 1: The four camera setup in this work and their four different points of view.

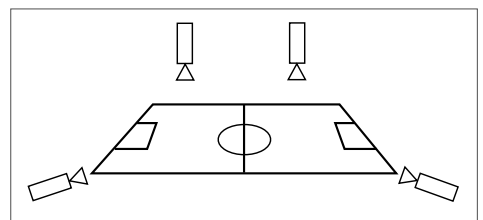

Figure 2: The camera positioning around the indoor soccer court. Each camera's field of view is set up to cover half a court allowing an overlapping in the center region of the court. In this setup, each player is covered by, at least, two cameras.

a map of the soccer court containing some points of interest such as the penalty marks, the center and the corners. The coordinates in such interest points are important to allow us to find a homography matrix mapping image coordinates to world coordinates. For each camera, we need to find a transformation to lead the detections in the image coordinates to the world coordinates we are interested in.

The contribution of this paper relies on the approach we use to project the different detections from different cameras (which are in image coordinates) to world coordinates and fuse them in order to take advantage of the multiple cameras and subsequent redundant information. For this, we represent the indoor soccer court as a bidirectional and multimodal probability function of a given player be detected in a given position. For fusion, each projected point is transformed in a Gaussian and the player positions in world coordinates are found by means of a gradient ascent algorithm.

The rest of this paper is organized as follows. Section 2 shows the related methods for detecting objects in images with an emphasis on the most cited works . Section 3 presents the details of our contribution and explains its different stages: observation, projection and localization. Section 4 shows comparative experiments and results using the proposed approach with respect to a classical method in computer vision with the highest number of citations in the last 10 years. Finally, Section 5 wraps up the paper and discusses future directions of the work.

\section{Related Methods}

Automatic people detection in images is a problem widely investigated by the scientific community. The reason is the high number of possible applications such as security and monitoring environments and pedestrian counting. Image analysis techniques as a tool for aiding team sports such as indoor soccer have also increased in the last years raising the interest of the scientific community for developing better tools and solutions.

The literature presents some works in this line with the same objectives. We have found detection of objects of interest (players) by means of background subtraction using approaches (Kang et al., 2003; Hamid et al., 2010; Ming et al., 2009; Khan and Shah, 2006) as the work of Stauffer and Grimson (Stauffer and Grimson, 1999), or by using color histograms to eliminate the predominant colors (Tong et al., 2011). Another approach uses a fixed background model calculated periodically (Figueroa et al., 2006). The work of Alahi et al. (Alahi et al., 2009) performs detection of basketball players based on silhouettes in omnidirectional cameras.

In some cases, the detection needs adjustments, like shadows elimination (Hamid et al., 2010). In that case, the authors use homograph to project image blobs onto other cameras and eliminate pixels with similar values. Kang et al. (Kang et al., 2003) also use homograph, however, the objective is to construct a global map of probability for the foreground. Some works also use the localized positions to integrate trajectories (Alahi et al., 2009; Khan and Shah, 2006), others use Kalman Filters (Hamid et al., 2010; Kang et al., 2003) or Markov Chain Monte Carlo (MCMC) to perform tracking (Tong et al., 2011). Some works present approaches based on graphs to determine positions and track objects (Hamid et al., 2010; Figueroa et al., 2006).

Different from previous approaches, according to the Computer Vision point of view, we can handle the problem of detecting players in a game as an object detection problem in which each player is an object to be detected. In this sense, 
Viola and Jones (Viola and Jones, 2001) proposed a real time object detection method that represents a breakthrough in the computer vision research in the last 10 years. The original work was focused on face detection but the extension to different objects (e.g., people) is straightforward.

Complementing the vision approach represented by Viola and Jones methods, Felzenszwalb et al. (Felzenszwalb et al., 2010) introduced another method for object detection based on multi-scale deformable parts. Similar to Viola and Jones' method, this approach also requires a training set with positive and negative examples of the object of interest. The method decomposes the objects into multiple scales and capture local appearance details of the objects of interest while connecting the parts by means of a deformable model.

Two questions with respect to the previous approaches are: how to perform the object detection without the need for background separation and also how to take advantage of multiple camera detections at relative low computational cost. In this paper, rather than using color information or background subtraction operations, we use a simple and well known Viola and Jones (Viola and Jones, 2001) object detector method for detecting objects of interest (e.g., the players) in each camera. This method works based on patterns present in the objects instead of color information and quickly finds objects in the images. In addiction, we propose a new form for combining observations from multiple different cameras (in our case four cameras) taking advantage of possible redundant information.

\section{Indoor Soccer Player Detection from Multiple Cameras}

In this section, we present our approach for indoor soccer player detection from multiple cameras. The approach is divided into three stages as Figure 3 depicts. We present each stage in more details in the next sections.

1. Stage \#1 independently detects the players in the image plane of each camera. This stage can use any object detector trained for detecting indoor soccer players. In this paper, we use the Viola and Jones (Viola and Jones, 2001) detector.

2. Stage \#2 projects the observed objects (players) of the previous stage onto a plane representing world coordinates. We refer to this plane as world plane as it is a representation of the actual soccer court.

3. Stage \#3 combines the different projections using a bidimensional multimodal probability function representing the potential player positions in the soccer court. It employs a gradient ascent algorithm to find the most probable soccer player candidate positions among the different observations in the world plane.

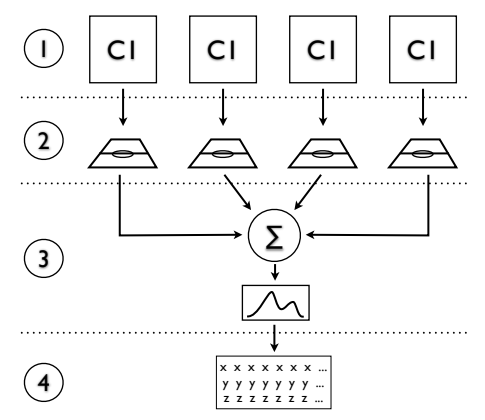

Figure 3: The proposed approach and its three stages. (1) independent detection; (2) projection of the observations from the image plane to the world plane; (3) representation of the observation in a multimodal bidirectional probability function and determination of the most probable positions (numbers 3 and 4 in the figure).

\subsection{Stage \#1 - Independent Image Plane Observations}

To detect the indoor soccer players from multiple cameras, we first need to independently detect the players in the image plane of each camera and build an observation model. For this task, we can use several different object detectors such as (Viola and Jones, 2001; Felzenszwalb et al., 2010). In this paper, we use the Viola and Jones (Viola and Jones, 2001) object detector trained with indoor soccer players. This is a classical method with high-citation count in the computer vision literature. In addiction, it has a simple training (though relatively slow), it is open-source and also with no patents attached.

\section{The Viola and Jones Detector}

Viola and Jones (Viola and Jones, 2001) presented an approach based on Haar-filters and on an Adaboosting machine learning algorithm to detect objects in images. Initially, the authors focused on face detection. The extension of the 
detector to other types of objects (e.g., people) is straightforward. For that, the requirement is to obtain enough training examples representing people vs. non-people images for a new training procedure.

The Viola and Jones detector relies on simple features known as Haar-like features. A Haar-like feature is calculated using sums and differences as Figure 4 depicts.

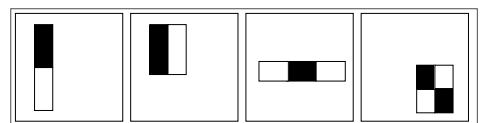

Figure 4: Example of Haar-features of two, three and four rectangles. The value of a feature is given by the difference of the sum of pixel values in the regions with different colors. In this case, the value of the feature is given by the difference between the black and white region.

The method uses sliding windows of size $24 \times$ 24 pixels to detect faces. Within each window, there are more than 180,000 possible features with different sizes and orientations. For fast sum and difference calculations, the authors propose the concept of integral images. An integral image is an image with the same dimensions of the original image but each point represents the sum of every pixel above and to the left of the current pixel position

$$
i i(x, y)=\sum_{x^{\prime} \leq x, y^{\prime} \leq y} i\left(x^{\prime}, y^{\prime}\right) .
$$

We can calculate the integral image $i i$ with only one pass over each image pixel. With this integral image, we can calculate the summation of a given rectangle feature with only three accesses to the integral image.

The authors propose to view the Haar-like features as weak classifiers. For that, they use the Adaboost algorithm to select the best features among the more than 180,000 possible ones. For each weak classifier, the Adaboosting training algorithm determines the best threshold that results the lowest classification error for object and non-object classes. A weak classifier $h_{j}(x)$ is given by a feature $f_{j}$, a threshold $\theta_{j}$ and a polarization $(+/-) p_{j}$

$$
h_{j}(x)= \begin{cases}1 & \text { if } p_{j} f_{j}(x)<p_{j} \theta_{j} \\ 0 & \text { otherwise. }\end{cases}
$$

where $x$ is a $24 \times 24$-pixel image region. For each weak classifier selection, the most appropriate feature is selected and receives a weight associated with each training sample evaluated.
With several weak classifiers, it is possible to combine them to build a strong classification procedure. The authors propose this combination using a cascade setup. In a cascade classifier scheme, the outcome of one classifier is the input the next one giving rise to a strong classifier as Figure 5 depicts.

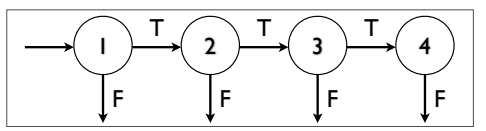

Figure 5: Viola and Jones (Viola and Jones, 2001) cascade of classifiers. Each weak classifier classifies such sub image to detect whether or not it has the object of interest. If a sub image passes over all the classifiers, it is tagged as having the object of interest.

\subsection{Stage $\# 2$ - Observation Projection onto the World Plane}

The result of each detector for each camera represents object observations in the image world for each camera. However, we are interested in the soccer player localization in the world plane that represents the actual soccer court in which the players are. The world plane is represented in $3 \mathrm{D}$ coordinates. As we mentioned before, we have some control points in the soccer court whose location we know a priori (e.g., penalty marks and corner marks). With such points, we can use a video frame in a camera to find such correspondences manually.

The homography maps the coordinates between the planes. In our case, the objects of interest move on the soccer court and, therefore, are always on a plane in the $3 \mathrm{D}$ world. We can use the homography of specific points of the object detections (e.g., the foot of a player) to find their localization in the world coordinates.

Each player as found by a detector is represented by a rectangle in the image plane of a given camera. In our work, we consider the midpoint of the basis of such rectangle as a good representation of a player's feet in the image plane. As we expect, the estimation of the player's feet position is not perfect and consequently its projection to the world coordinates does not represent the exact point in which the player is at. In addition the the detector error, the homography also contains intrinsic errors.

After the projection, we can have more than one point associated with the same player and we need a fusion approach to better estimate the 
player positions taking advantage of the multiple camera detections.

\subsection{Stage \#3 - Multiple Camera Fusion}

After the detection of the players from multiple cameras, we have a set of observations in the image plane of each camera (each rectangle represents the detection of a player in a given camera). Assuming that the midpoints in the base of each rectangle is a good choice for the localization of the players' feet, we project such midpoints onto the world plane (representing the soccer court) using the homography matrix related to the camera under consideration.

Due to detection as well errors in projections, these points do not correspond to the exact localization of the players. However, the projected points are a good estimation for the player's localization in a region.

With possibly more than one detection per player as well as with possible projection errors, the question is how to best estimate the players' position. This paper's contribution goes in this direction. For this, we represent the world plane (soccer court) as a mixture of Gaussians whose parameters vary according to the source camera of a projection. The parameters (mean and covariance) of a Gaussian function for each camera can be calculated from one or two short video sequences serving as training examples.

For finding the Gaussian parameters, we can use annotated training sequences with the players' positions marked with the assistance of a human. These annotations are used to measure the error related to the projections of each point onto the world plane. With this, we calculate the average error in $x$ and $y$ directions and the covariance matrix for the camera projection to represent each point projection as a Gaussian function.

To calculate such measures, we need to make the linking among the annotated points and the detected points. We assume a projected point corresponds to the closest annotated point in the world plane. In some cases, for instance when several players are close together, this assumption is not good. To alleviate this, during training, we choose a training sequence in which the players are reasonably separated in the soccer court. This means that a detection has one closest point easily identifiable and its second closest point is relatively distant. We choose all the cases in which the closest correspondences to the annota- tions are within a radius $L_{1} \leq 2$ meters and the second closest correspondences are farther than $L_{2} \geq 3$ meters.

With this representation, each projected point is represented as a 2D Gaussian center in the position of the projected point corrected by the average error and the calculated covariance matrix corresponding to the source camera in question. With all points represented the same way, we have a unique function representing the entire soccer court which gives us the probability of the position of each player in the court. Figure 6 depicts an example of such function.

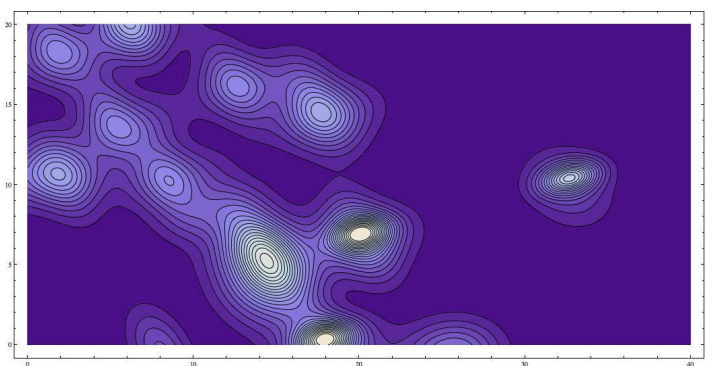

Figure 6: 2D probability function example. The multiple camera projections are replaced by Gaussian functions with parameters obtained during training. This function gives the probability of having a player in a given position (represented with contours in this figure).

Figure 7 depicts one case with several players relatively close to one another. In this case, the corresponding Gaussian functions are also close together. Consequently, close peaks are merged. The reason is that the covariance matrices yield wide base Gaussians. To diminish such effects, we can multiply the covariance matrices for a fixed scale factor resulting in a multimodal function with well defined peaks. In this paper, we fix such a scale parameter in 0.2 for all experiments.

For analysis of new sequences, a probability function is formed for each new video frame based on the projections from multiple cameras. The most probable positions correspond to the realworld positions likely to contain players and are equivalent to the peaks represented in Figure 6 .

\section{Determining the Player Positions Using Gradient Ascent}

With the devised function, we need a method to find the closest peak of a projected point. For this, we can use a simple gradient ascent algorithm. In a mixture of Gaussians, the gradient of the composite function is given by the vector 

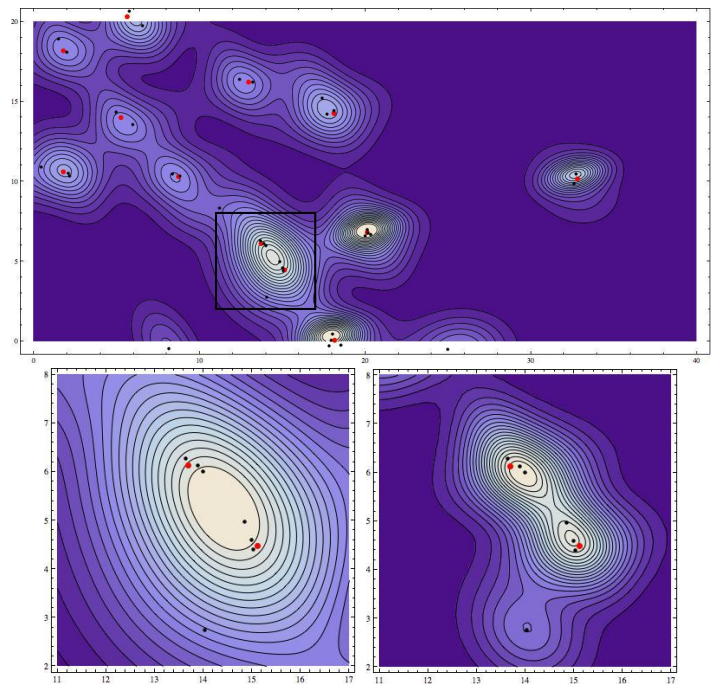

Figure 7: Contour around the points of interest. Red dots represent ground truth annotations. Black dots represent projections from the four cameras with no distinction. On top, we have the whole function surface. On the left, we have a zoom in the selected region with players close together. On the right, we have the same region with the covariance matrices multiplied by a fixed scale factor of 0.2 .

sum of the partial gradients. We can calculate the gradient as in Eq. 3

$$
\nabla F=\nabla G_{1}+\nabla G_{2}+\ldots+\nabla G_{n} .
$$

The partial gradients need to be calculated separately. Let $\Sigma$ be a covariance matrix, $\mu$ the average and $X$ the point for which we are interested in calculating the probability.

$$
\Sigma=\left[\begin{array}{ll}
\sigma_{1} & \sigma_{2} \\
\sigma_{3} & \sigma_{4}
\end{array}\right], X=\left[\begin{array}{l}
x \\
y
\end{array}\right], \mu=\left[\begin{array}{l}
\mu_{x} \\
\mu_{y}
\end{array}\right] .
$$

The Gaussian function $G(X)$ is given by

$$
G(X)=\frac{1}{\sqrt{2 \pi|\Sigma|}} e^{-\frac{1}{2}[X-\mu]^{T} \Sigma^{-1}[X-\mu]},
$$

where we find the exponent $B$ and the factor $F$ according to

$$
B=-\frac{1}{2}[X-\mu]^{T} \Sigma^{-1}[X-\mu] \quad \text { e } \quad F=\frac{1}{\sqrt{2 \pi|\Sigma|}}
$$

allowing us to re-write Eq. 5 as

$$
G(X)=F e^{B},
$$

and its gradient as

$$
\nabla G(X)=\left[F e^{B} \frac{d B}{d x}, F e^{B} \frac{d B}{d y}\right] .
$$

The derivative $\frac{d B}{d x}$ depends on the inverse of the covariance matrix $\Sigma^{-1}$. In our case, we have a $2 \times 2$ covariance and by using the adjoint matrix we obtain the inverse

$$
\begin{aligned}
\Sigma^{-1} & =\frac{1}{|\Sigma|} \cdot \operatorname{adj}(\Sigma) \\
& =\frac{1}{|\Sigma|} \cdot\left[\begin{array}{cc}
\sigma_{4} & -\sigma_{2} \\
-\sigma_{3} & \sigma_{1}
\end{array}\right] .
\end{aligned}
$$

Replacing Eq. 9 into Eq. 6, we obtain:

$$
B=-\frac{1}{2 \cdot|\Sigma|}\left(x^{2} \sigma_{4}+y^{2} \sigma_{1}-x y\left(\sigma_{3}+\sigma 2\right)\right)
$$

and hence the derivatives in $x$ and $y$ axis are

$$
\begin{aligned}
& \frac{d B}{d x}=-\frac{1}{2 \cdot|\Sigma|}\left(2 x \sigma_{4}-y\left(\sigma_{3}+\sigma_{2}\right)\right) \\
& \frac{d B}{d y}=-\frac{1}{2 \cdot|\Sigma|}\left(2 y \sigma_{1}-x\left(\sigma_{3}+\sigma_{2}\right)\right)
\end{aligned}
$$

We can find the players' positions by looking for the local maxima following the gradient. Each projected point onto the world plane converges to its closest peak according to this representation. As more than one camera can detect the same player it is reasonable to assume that the points representing such detections converge to the same peak. After the analysis, each peak corresponds to the most likely players' positions in the court.

\section{Experiments and Methodology}

Our method consists of a training phase responsible for calibrating the parameters representing the problem in question and a testing phase responsible for finding the players taking advantage of multiple cameras.

We use seven Full-HD games each one shot with four cameras according to the camera setup depicted in Figure 2. Each game has the first and second times (common in soccer games). For simplicity, we consider each time as a game. Therefore, we have 14 games in total.

The games were shot during the 2009 South American Women Indoor Soccer Championship that took place in Brazil. All the videos were resampled at $720 \times 480$ pixels.

We separate one of the 14 games (4 videos) for training and parameter calculation as described in the previous section. One time of a game consists of 20 minutes of shooting per camera at 30 frames per second $(36,000$ frames). The remaining 13 games $(4 \times 13$ videos $)$ were used for testing.

To compare the detections and evaluate their quality, we annotate the real players' positions on the videos by hand for each frame. We used the method proposed by (Figueroa et al., 2006) for 
aiding in the annotation process an construct a baseline. We have ground truth annotations for the 10 players plus the two referees for one minute for each of the games. The game used for training was entirely annotated (20 minutes).

The first step of the proposed method consists of training the object detectors for finding soccer players. This training was performed using positive and negative samples from the training video sequence. The set of positive samples consists of rectangles around players as seen by the four cameras. We used approximately 16,000 positive samples. The negative samples consist of any rectangle not containing a player. We used approximately 18,000 negative samples.

After the training of the detector, we perform the training of the proposed approach for calibrating the parameters related to the multimodal function representing the soccer court as we discussed in Section 3.3. As we mentioned, we used the first game sequence for this intent.

We compare our method to the detection using each isolated camera using the standard Viola and Jones (Viola and Jones, 2001) with no fusion. For each detection, we calculate the Euclidean distance to the annotation representing the real player's position. The smaller the distance the better the detection.

When we use only the detectors separately, we project the detections in the image plane of one camera onto the world coordinates and each projection is treated independently. When we use the proposed approach, all the detections from the four different cameras are projected onto the world plane and the points are combined using the proposed multimodal function and gradient ascent method. In both cases, each point in the end is compared to the closest annotated point for determining the detection error.

The distance between an actual position and a detection measures the estimation error and it is measured in meters. Figures 8 and 9 show the best and worst case when comparing both detection approaches (with and without multiple camera fusion). In both cases, we show the average error in each testing frame. We calculate the error for each camera separately given that the projection errors are different for each camera.

Table 1 shows the final average errors and standard deviation per video per camera. In most cases our approach improves the players' localization compared to the approach with no fusion demonstrating the potential of fusion for helping further analysis such as tracking of players.

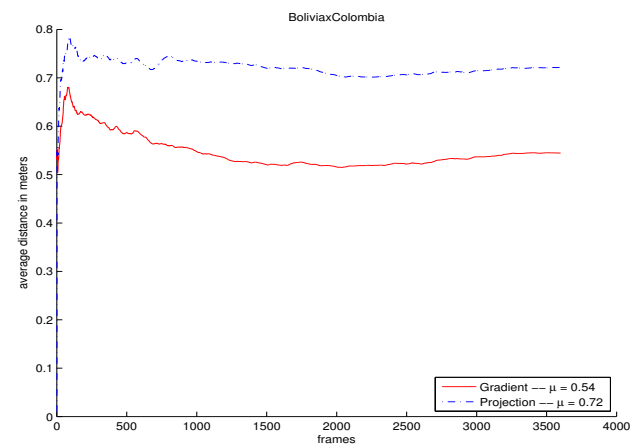

Figure 8: Estimation average error. The curve shows the average error across the concatenated frames of different test video sequences considering one camera. The figure shows one case in which our approach is significantly better than the approach with no fusion.

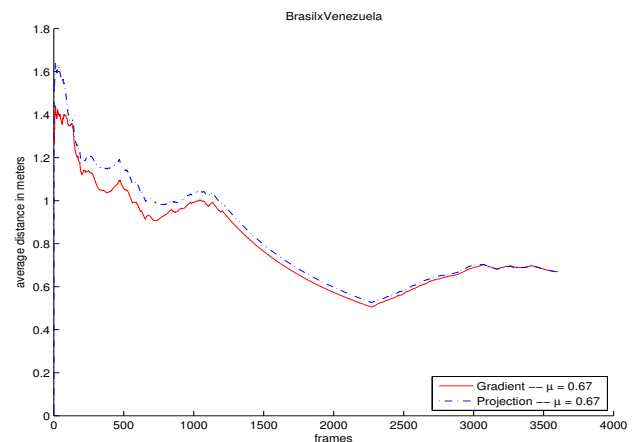

Figure 9: Estimation average error. The curves show the average error across the concatenated frames of different test video sequences considering one camera. The figure shows a case in which both detection approaches are not statistically different.

\section{Conclusions}

In this paper, we presented an approach for estimating the players' positions in all moments of indoor soccer games. For that, we observe stationary cameras set up around the soccer court.

The obtained results show the potential of the proposed approach as it reduces the error of the detected position of players and represents a possible aid for further tasks such as tracking the players.

The proposed approach uses a simple object detector for each camera and projects different detections onto a world plane representing the soccer court. The approach then fuses the observations by means of a bidimensional multimodal function with parameters calculated during training. The training is fairly simple and requires only one video sequence per camera. The best players' positions are given by a gradient ascent 
Table 1: Average error and standard deviation per camera.

\begin{tabular}{l|c|c|c|c} 
& \multicolumn{2}{|c|}{ Fusion } & \multicolumn{2}{c}{ No Fusion } \\
Game & $\mu$ & $\sigma$ & $\mu$ & $\sigma$ \\
\hline BoliviaxColombia-c0-t1 & 0.58 & 0.13 & 0.73 & 0.16 \\
BoliviaxColombia-c1-t1 & 0.68 & 0.19 & 0.78 & 0.20 \\
BoliviaxColombia-c2-t1 & 0.75 & 0.24 & 0.88 & 0.21 \\
BoliviaxColombia-c3-t1 & 0.53 & 0.14 & 0.65 & 0.15 \\
BoliviaxColombia-c0-t2 & 0.65 & 0.21 & 0.77 & 0.21 \\
BoliviaxColombia-c1-t2 & 0.76 & 0.18 & 0.91 & 0.19 \\
BoliviaxColombia-c2-t2 & 0.54 & 0.13 & 0.72 & 0.13 \\
BoliviaxColombia-c3-t2 & 0.76 & 0.20 & 0.91 & 0.20 \\
BrasilxArgentina-c0-t1 & 0.71 & 0.21 & 0.83 & 0.20 \\
BrasilxArgentina-c1-t1 & 0.74 & 0.27 & 0.79 & 0.27 \\
BrasilxArgentina-c2-t1 & 0.72 & 0.24 & 0.81 & 0.24 \\
BrasilxArgentina-c3-t1 & 0.55 & 0.16 & 0.63 & 0.15 \\
BrasilxArgentina-c0-t2 & 0.84 & 0.27 & 0.91 & 0.25 \\
BrasilxArgentina-c1-t2 & 0.63 & 0.19 & 0.68 & 0.19 \\
BrasilxArgentina-c2-t2 & 0.82 & 0.24 & 0.90 & 0.23 \\
BrasilxArgentina-c3-t2 & 0.79 & 0.25 & 0.89 & 0.23 \\
BrasilxColombia-c0-t1 & 0.78 & 0.21 & 0.82 & 0.20 \\
BrasilxColombia-c1-t1 & 0.81 & 0.23 & 0.90 & 0.23 \\
BrasilxColombia-c2-t1 & 0.78 & 0.25 & 0.90 & 0.22 \\
BrasilxColombia-c3-t1 & 0.80 & 0.21 & 0.84 & 0.21 \\
BrasilxColombia-c0-t2 & 0.81 & 0.22 & 0.87 & 0.19 \\
BrasilxColombia-c1-t2 & 0.77 & 0.21 & 0.81 & 0.19 \\
BrasilxColombia-c2-t2 & 0.88 & 0.27 & 1.00 & 0.26 \\
BrasilxColombia-c3-t2 & 0.67 & 0.19 & 0.79 & 0.19 \\
BrasilxPeru-c0-t1 & 1.10 & 0.35 & 1.12 & 0.32 \\
BrasilxPeru-c1-t1 & 0.66 & 0.19 & 0.73 & 0.20 \\
BrasilxPeru-c2-t1 & 0.64 & 0.20 & 0.74 & 0.19 \\
BrasilxPeru-c3-t1 & 1.06 & 0.36 & 1.10 & 0.30 \\
\hline & & & &
\end{tabular}

algorithm applied over the calculated Gaussian function. The results show the detections are only a few centimeters off their real positions with small standard deviation and improves the detection when compared to an approach with no fusion.

Future work includes performing the tracking of the players. For that we intend to use the multimodal probability function as an observation model for a particle filter allowing us to consistently track the players using multiple cameras.

\section{REFERENCES}

Alahi, A., Boursier, Y., Jacques, L., and Vandergheynst, P. (2009). Sport player detection and tracking with a mixed network of planar and omnidirectional cameras. In ICDSC.

Felzenszwalb, P., Girshick, R., McAllester, D., and Ramanan, D. (2010). Object detection with discriminatively trained part based models. PAMI, 32(9):1627-1645.

Figueroa, P., Leite, N., and Barros, R. (2006). Tracking soccer players aiming their kinematical motion analysis. CVIU, 101(2):122-135.

\begin{tabular}{l|c|c|c|c} 
& \multicolumn{2}{|c|}{ Fusion } & \multicolumn{2}{c}{ No Fusion } \\
Game & $\mu$ & $\sigma$ & $\mu$ & $\sigma$ \\
\hline BrasilxPeru-c0-t2 & 0.97 & 0.25 & 1.03 & 0.22 \\
BrasilxPeru-c1-t2 & 0.89 & 0.23 & 0.93 & 0.22 \\
BrasilxPeru-c2-t2 & 0.98 & 0.23 & 1.01 & 0.21 \\
BrasilxPeru-c3-t2 & 0.91 & 0.22 & 0.96 & 0.19 \\
BrasilxVenezuela-c0-t1 & 0.50 & 0.15 & 0.66 & 0.17 \\
BrasilxVenezuela-c1-t1 & 0.58 & 0.25 & 0.82 & 0.24 \\
BrasilxVenezuela-c2-t1 & 0.36 & 0.18 & 0.53 & 0.17 \\
BrasilxVenezuela-c3-t1 & 0.45 & 0.14 & 0.52 & 0.14 \\
BrasilxVenezuela-c0-t2 & 0.81 & 0.64 & 0.85 & 0.65 \\
BrasilxVenezuela-c1-t2 & 0.95 & 0.59 & 0.89 & 0.61 \\
BrasilxVenezuela-c2-t2 & 0.79 & 0.61 & 0.83 & 0.64 \\
BrasilxVenezuela-c3-t2 & 0.67 & 0.67 & 0.67 & 0.68 \\
ColombiaxUruguai-c0-t1 & 0.90 & 0.27 & 1.04 & 0.25 \\
ColombiaxUruguai-c1-t1 & 0.77 & 0.24 & 0.93 & 0.23 \\
ColombiaxUruguai-c2-t1 & 0.56 & 0.16 & 0.65 & 0.16 \\
ColombiaxUruguai-c3-t1 & 0.74 & 0.25 & 0.71 & 0.23 \\
ColombiaxUruguai-c0-t2 & 0.73 & 0.16 & 0.89 & 0.17 \\
ColombiaxUruguai-c1-t2 & 0.78 & 0.19 & 0.86 & 0.21 \\
ColombiaxUruguai-c2-t2 & 0.86 & 0.24 & 0.93 & 0.23 \\
ColombiaxUruguai-c3-t2 & 0.63 & 0.16 & 0.62 & 0.13 \\
PeruxBolivia-c0-t1 & 0.68 & 0.25 & 0.77 & 0.25 \\
PeruxBolivia-c1-t1 & 0.79 & 0.25 & 0.81 & 0.25 \\
PeruxBolivia-c2-t1 & 0.94 & 0.30 & 1.03 & 0.28 \\
PeruxBolivia-c3-t1 & 0.77 & 0.35 & 0.87 & 0.30 \\
PeruxBolivia-c0-t2 & 0.71 & 0.23 & 0.80 & 0.24 \\
PeruxBolivia-c1-t2 & 0.84 & 0.26 & 0.80 & 0.24 \\
PeruxBolivia-c2-t2 & 0.93 & 0.29 & 1.00 & 0.32 \\
PeruxBolivia-c3-t2 & 0.80 & 0.28 & 0.86 & 0.26 \\
\hline & & & &
\end{tabular}

Hamid, R., Kumar, R., Grundmann, M., Kim, K., Essa, I., and Hodgins, J. (2010). Player localization using multiple static cameras for sports visualization. In $C V P R$, pages 731-738.

Kang, J., Cohen, I., and Medioni, G. (2003). Soccer player tracking across uncalibrated camera streams. In VS-PETS, pages 172-179.

Khan, S. and Shah, M. (2006). A multiview approach to tracking people in crowded scenes using a planar homography constraint. In ECCV.

Ming, Y., Guodong, C., and Lichao, Q. (2009). Player detection algorithm based on gaussian mixture models background modeling. In ICINIS

Stauffer, C. and Grimson, W. (1999). Adaptive background mixture models for real-time tracking. In CVPR, volume 2, pages $252-260$.

Tong, X., Liu, J., Wang, T., and Zhang, Y. (2011). Automatic player labeling, tracking and field registration and trajectory mapping in broadcast soccer video. ACM TIST, 2(2).

Viola, P. and Jones, M. (2001). Rapid object detection using a boosted cascade of simple features. In $C V P R$, volume 1 , pages $511-518$. 